\title{
Ultrasonic Inspection System Using a Long Waveguide with an Acoustic Horn for High-Temperature Structure
}

\author{
Riichi Murayama1, Masayuki Kobayashi', Kenshi Matsumoto', Makiko Kobayashi² \\ ${ }^{1}$ Department of Intelligent Mechanical Engineering, Fukuoka Institute of Technology, Fukuoka, Japan \\ ${ }^{2}$ Department of Computer Science and Electrical Engineering, Kumamoto University, Kumamoto, Japan \\ Email: kobayashi@cs.kumamoto-u.ac.jp, murayama@fit.ac.jp
}

Received 31 August 2014; revised 30 September 2014; accepted 31 October 2014

Copyright (C) 2014 by authors and Scientific Research Publishing Inc.

This work is licensed under the Creative Commons Attribution International License (CC BY).

http://creativecommons.org/licenses/by/4.0/

(c) (i) Open Access

\begin{abstract}
The nondestructive inspection of a high temperature structure is required in order to guarantee its safety. However, there are no useful sensors for high temperature structures. Some of them cannot work at temperatures over $50^{\circ} \mathrm{C}$. Another concern is that they are too expensive to use. A sensing system, which can transmit and receive an ultrasonic wave that travels a long distance using a long waveguide, has been studied. We confirmed that the optimal guided ultrasonic wave could travel more than $10 \mathrm{~m}$ using an electromagnetic transducer (EMAT) with a thin Ni-sheet surrounded on the surface of the bar and a 2-mm diameter bar as the waveguide. However, we had the difficult problem of receiving the reflected ultrasonic wave from the inside of a test specimen. We tried to improve the trial inspection system using an acoustic horn. An experiment in which the temperature of the test block was heated to about $500^{\circ} \mathrm{C}$ has now been completed. Finally, the condition of the bend in the waveguide to pass without reflection was confirmed.
\end{abstract}

\section{Keywords}

Guide Wave, Waveguide, Acoustic Horn, NDI, Thickness Measurement

\section{Introduction}

Ultrasonic methods employing piezoelectric ultrasonic transducers (UTs) are widely used for the real-time, in situ or off-line non-destructive evaluation (NDE) of large metallic and polymeric composite structures including airplanes, automobiles, ships, pressure vessels, and pipelines. Their advantage includes a subsurface and inner inspection capability, high inspection speed, simplicity, and cost-effectiveness. Common limitations of the cur- 
rent UTs are the requirement of a couplant and their difficult use at high temperatures (HTs). However, for the in-line monitoring of industrial processes and the NDE of the structures mentioned above, UTs are often required to operate at HTs [1] [2]. However, there are no useful sensors for a high-temperature structure. Some of them cannot work at temperatures over $50^{\circ} \mathrm{C}$. Another concern is that they are too expensive to use. Therefore, the sensing system, which can transmit and receive an ultrasonic wave that can travel a long distance using a long waveguide, has been studied. The developed system suggests that an ultrasonic sensor could work at atmosphere temperature for a long time.

\section{Basic Idea for the Measurement of a High-Temperature Structure}

There are many sensing systems including an ultrasonic wave sensor for inspecting a normal temperature structure. However, it is difficult to use a general ultrasonic wave sensor for high-temperature structure measurements. The non-contact sensor, which is applicable for high temperature structures, includes an electromagnetic ultrasonic acoustic transducer (EMAT) using an electromagnetic force. However, it requires a cooling system which has many problems. In addition, there is the laser ultrasonic wave, which is a non-contact sensor, but its performance is unstable and very expensive. As shown in Figure 1, this sensing system transmits and receives an ultrasonic wave from a long distance using a long waveguide which is a bar with a small diameter. As a result, an ultrasonic sensor could be used at ambient temperature. Actually, a guide wave in a bar, the L(1) mode, as one of the ultrasonic modes was selected because the distance attenuation characteristic, which shows a decrease in an ultrasonic wave as it travels in a bar, was said to be lower than any other ultrasonic wave modes, for example, a longitudinal wave.

\section{Temperature Influence Analysis of the Wave Guide}

The temperature distribution along a bar was calculated in order to determine the length of a wave guide which could be used in the preliminary experiment. As the temperature of the high-temperature structure, $600^{\circ} \mathrm{C}$ was assumed and evaluated. Figure 2 shows the analysis model. The temperature distribution along a bar was analysed using this analysis model as the heat transfer of a fin. For the thermal conductivity conditions of iron: $\lambda=80.3\left[\mathrm{~W} /\left(\mathrm{m}^{2} \cdot \mathrm{k}\right)\right]$, aerial heat transfer rate $\alpha=50\left[\mathrm{~W} /\left(\mathrm{m}^{2} \cdot \mathrm{k}\right)\right]$, temperature at the edge of a bar: $\mathrm{T}_{0}=600\left[{ }^{\circ} \mathrm{C}\right]$, and temperature in the atmosphere: $T_{b}=20\left[{ }^{\circ} \mathrm{C}\right]$, the analysis solution becomes expression (1),

$$
\theta=\frac{\cosh [m(H-\chi)]+\frac{\alpha}{m \lambda} \sin h[m(H-\chi)]}{\cosh (m H)+\frac{\alpha}{m \lambda} \sin h[m H]}
$$

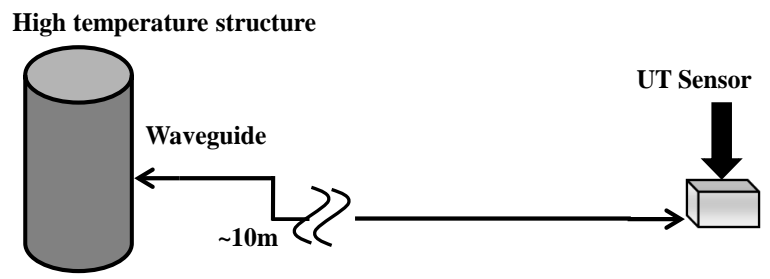

Figure 1. Basic idea using a long waveguide.

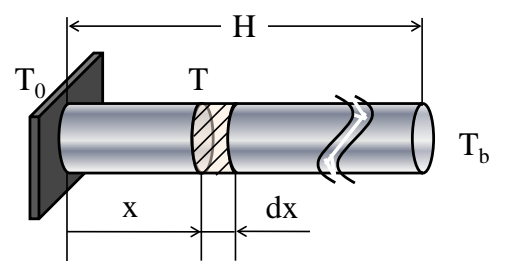

Figure 2. Analysis model. 
Here, $m=\sqrt{\alpha S /(\lambda A)}\left[\mathrm{m}^{-1}\right]$, bar length: $S[\mathrm{~m}]$, cross sectional area: $A\left[\mathrm{~m}^{2}\right]$, distance from the edge of the bar: $H[\mathrm{~m}]$, and distance to the heat transfer side: $x[\mathrm{~m}]$. The result analysed by Equation (1) is shown in Figure 3. As a result of the evaluation, if the length of the bar is over $500 \mathrm{~mm}$, the temperature at the opposite side of a bar is almost room temperature. Therefore, the basic preliminary experiment was done using the bar with a $500 \mathrm{~mm}$ length.

\section{Drive Principle of a Guide Wave in a Bar}

Generally, the oscillation component of a guide wave in a bar is mainly parallel to the axis direction under the conditions in which the wavelength is much longer than the diameter of the bar. This means that a guide wave sensor has to generate a force in which the vibration direction is parallel to the long direction of the bar. Actually, 3 types of ultrasonic sensors has been compared in order to meet our objective as shown in Figure 4. First, it is a method to generate a guide wave using the end face of the bar by the PZT-type as shown in Figure 4(a). A longitudinal wave was injected into the bar from the end surface and transformed into a guide wave. Next, we tried to vibrate the side surface of the bar using an EMAT. The EMAT uses the change in the magnetostriction as the drive force mainly in the case of ferromagnetic materials. A magnet was used to generate a static magnetic field in order to allow the maximum change in the magnetostriction. In addition, a high frequency electrical current was imposed on an electromagnetic induced coil in order to generate a dynamic magnetic field which is superimposed on the static magnetic field. The change in the magnetostriction is converted into an ultrasonic wave. On the other hand, the electromagnetic induced coil for the receiver could detect the ultrasonic wave based on the electromagnetic induction phenomenon [3]-[7]. The drive principle of an EMAT generating a guide wave in a bar is shown in Figure 4(b). The EMAT is composed of an electromagnet, which can generate a static magnetic field along the axis direction of the bar, and a circular-shaped sensor coil, which can generate a dynamic magnetic field along the axis direction of the bar. A compound magnetic field can oscillate along the axis direction of the bar, which is needed to generate a fundamental guide wave in the bar.

Finally, a method to use a thin Ni-sheet as a vibration source, actually, a thin Ni-sheet was attached by covering the side surface of the steel bar using adhesive as shown in Figure 4(c), adding the EMAT which is used as

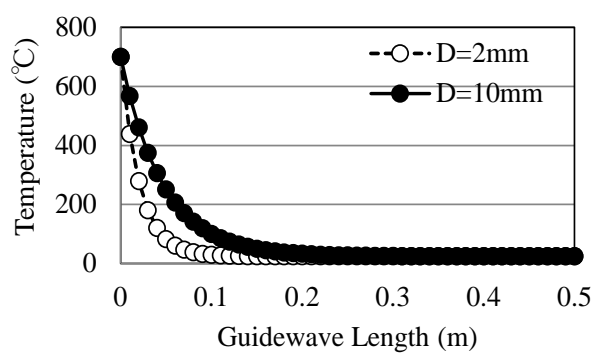

Figure 3. Temperature distribution of the waveguide.

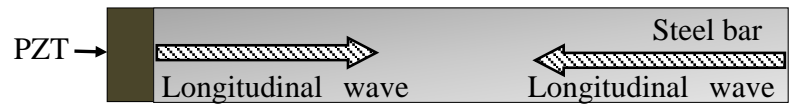

(a) Drive principle of the ultrasonic wave sensor using the PZT

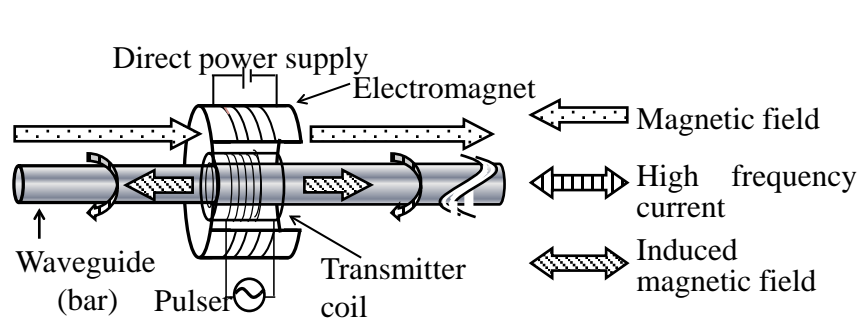

(b) Drive principle of a guide wave in a bar using EMAT

(Transmitter side)

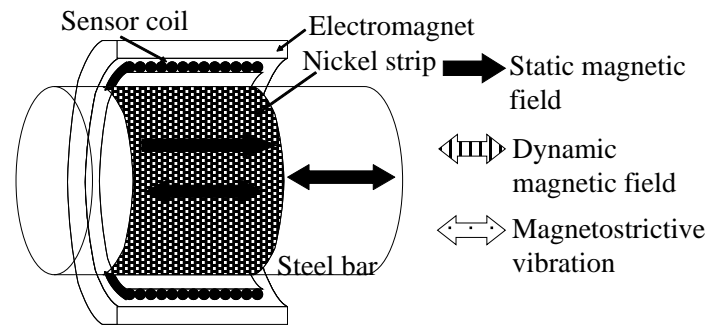

(C) Drive principle of a bar wave sensor using thin Nickel sheet

Figure 4. Drive principle of a guide wave using an EMAT. 
a drive force and a receiver as shown in Figure 4(b), because the magnetostriction of Ni was usually said to be greater than a that of a steel.

\section{Experimental System}

Figure 5 shows the experimental setup. A high frequency and power electrical signal of $200 \mathrm{~V}_{\mathrm{p}-\mathrm{p}}$ and up to 2 $\mathrm{MHz}$ was generated using a function generator which can inject a burst type-sine wave and a powered amplifier. A power supply of $40 V_{\max }$ and $3 A_{\max }$ was also used to drive the electromagnet. The received signal was amplified by a pre-amplifier of $40 \mathrm{~dB}$-amplification and a main amplifier of $60 \mathrm{~dB}$-amplification. The signal was observed by an oscilloscope and evaluated by a CPU.

\section{Experimental Results}

\subsection{Selection of the Waveguide}

We first tried to determine the specification of the steel bar used as the wave guide using the EMAT as shown in Figure 4(b). The reflected ultrasonic wave signals at the opposite side using many bars with $0.5-4.0 \mathrm{~mm}$ diameters and $500 \mathrm{~mm}$ length were measured to determine the most suitable diameter of the bar to be used as the waveguide, while changing their driving frequency from $200 \mathrm{kHz}$ to $1 \mathrm{MHz}$. Specifications of the electromagnet used in the experiment are shown in Table 1. Many trial sensors with different number of turns were fabricated to determine the best coil, which means that the highest received signal was detected. The specifications are shown in Table 2. As a result of the experiment, the transmitter coil with 10 turns and the receiver coil with 75 turns were selected for a $2 \mathrm{~mm}$ diameter bar.

The optimum drive frequency increased as the diameter decreased as shown in Figure 6(a). Next, the signal amplitude was the highest for a $2 \mathrm{~mm}$ diameter bar as shown in Figure 6(a). However, the distance attenuation was the lowest between 1.5 - $4.0 \mathrm{~mm}$ diameters for the bar as shown in Figure 6(b). As a result, the longest traveling distance, that was judged as the distance that the signal amplitude was 2 times higher than that of the noise signal, was achieved using a bar of 2-mm diameter and $200 \mathrm{kHz}$ drive frequency as shown in Figure 6(b).

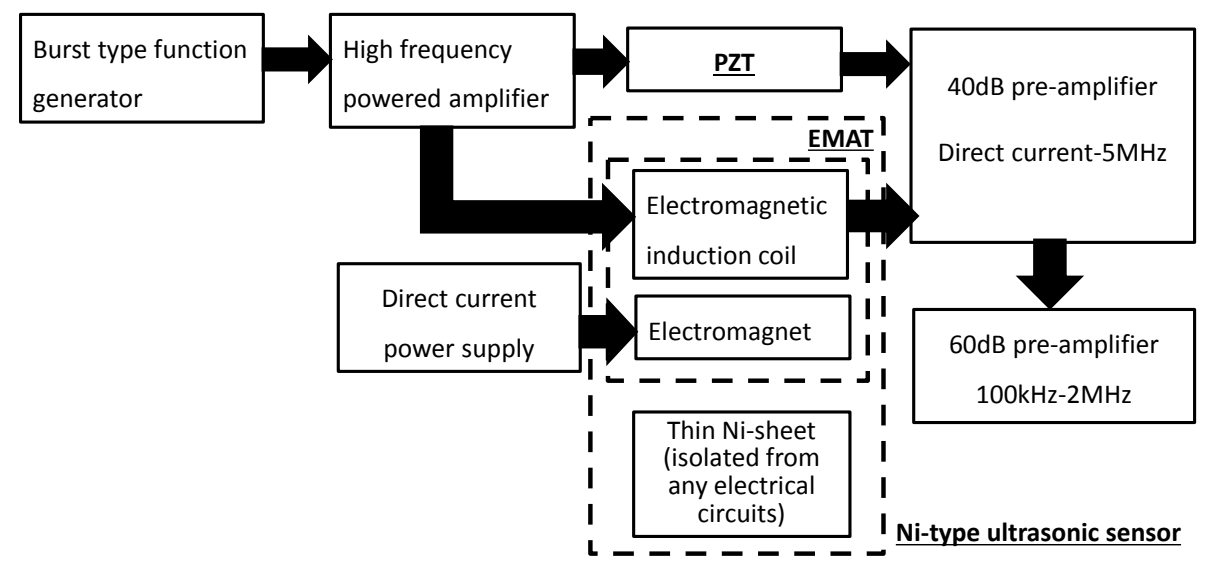

Figure 5. Experimental system.

Table 1. Specifications of the electromagnet.

\begin{tabular}{ccccc}
\hline$N[$ turn $]$ & $V[\mathrm{~V}]$ & $I[\mathrm{~A}]$ & $R[\Omega]$ & $H[\mathrm{~A} / \mathrm{m}]$ \\
\hline 400 & 4 & 5 & 0.8 & 8000 \\
\hline
\end{tabular}

Table 2. Number of turns of the sensor coil.

\begin{tabular}{ccccccc}
\hline & & \multicolumn{5}{c}{$N$ [Number of turns] } \\
\hline Transmitter & 3 & 5 & 10 & 20 & 100 & 120 \\
Receiver & 5 & 30 & 50 & 75 & 100 \\
\hline
\end{tabular}




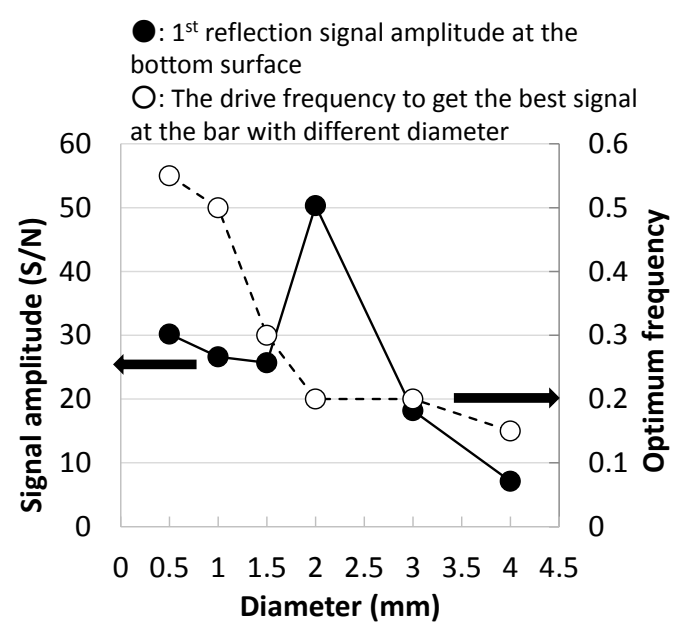

(a)

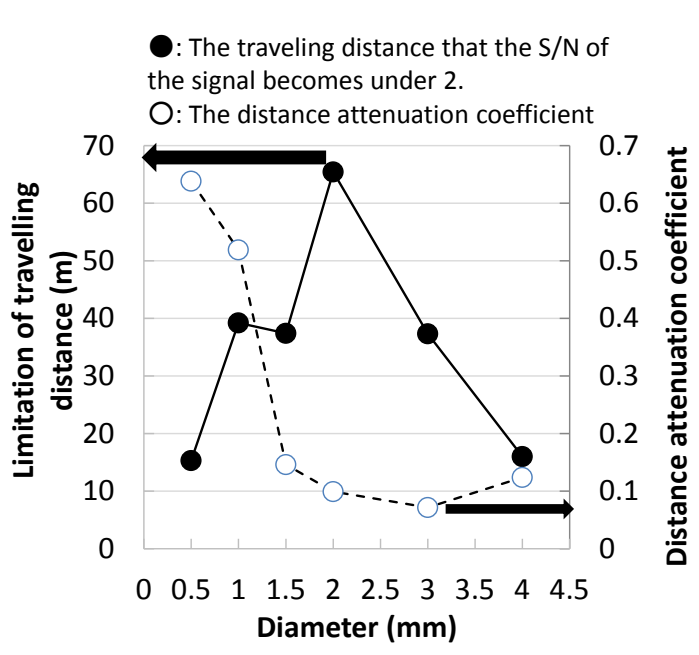

(b)

Figure 6. Relationship between the diameter of the waveguide and the received signal characteristic with different conditions. (a) Signal amplitude and optimum drive maximum travel distance; (b) Attenuation coefficient and the evaluated frequency.

\subsection{Selection of Ultrasonic Sensors}

We tried to select one of 3 types of ultrasonic sensors as shown in Figure 4. The experiment was conducted using the experimental system shown in Figure 5. The bar with a $2 \mathrm{~mm}$ diameter and $500 \mathrm{~mm}$ length and $200 \mathrm{kHz}$ drive frequency was used as the best condition which has been selected using the EMAT. A PZT element with 2 $\mathrm{mm}$ diameter, $10 \mathrm{~mm}$ thickness, and $200 \mathrm{kHz}$ optimum frequency was used to check the received signal. The element was attached on the end surface of the bar using an adhesive. The drive frequency was driven at 200 $\mathrm{kHz}$. The Ni sheet with a $30 \mathrm{~mm}$ length and $0.15 \mathrm{~mm}$ thickness was then attached at the position from the edge to $30 \mathrm{~mm}$, around the surface of the bar using an adhesive as the sensor in Figure 4(c). Finally, the 3 signals were compared as shown in Figure 7. The received signal by the Ni-sheet sensor was the highest among the 3 sensors and has a sharp waveform compared to the result by the PZT sensor.

Figure 8 shows the received ultrasonic wave signal reflected at the opposite side of the waveguide using the waveguides of $2 \mathrm{~m}$ and $10 \mathrm{~m}$ by the Ni-sheet sensor after optimization of the sensor. The $10 \mathrm{~m}$ waveguide was fabricated by welding five steel bars of $2 \mathrm{~m}$ each. The received signal with a good $\mathrm{S} / \mathrm{N}$ was detected even if the waveguide of a $10 \mathrm{~m}$ length was used as shown in Figure 8(b).

\subsection{Acoustic Horn for Thickness Measurement}

The attempt to detect the received ultrasonic signal reflected from the bottom of a test block of $100 \mathrm{~mm}$ thickness using a waveguide of $500 \mathrm{~mm}$ length is shown in Figure 9(a). The result is shown in Figure 9(b). The received signal reflected at the bottom of the test specimen must be detected between the $1^{\text {st }}$ reflected signal and the $2^{\text {nd }}$ reflected signal at the bottom edge of the waveguide, which means between $200 \mu$ s and $300 \mu$ s on the horizontal axis of Figure 9(b), if the performance of the sensor is sufficient to detect the signal. As a result, it was difficult to detect the reflected ultrasonic wave at the bottom surface of the test block using only the waveguide.

As the reason, the spread of the ultrasonic beam has been considered. As shown in Equation (2), which denotes the directivity angle of the ultrasonic beam, if the injection area of an ultrasonic wave is smaller, the angle of the beam spread increases. This means that the waveguide with the $2 \mathrm{~mm}$ diameter is too small as the ultrasonic wave source. According to Equation (2), the directivity angle becomes more than 360 degrees if the 200 $\mathrm{kHz}$ drive frequency and the $2 \mathrm{~mm}$ diameter bar were use. It turned out that it is necessary to use an acoustic horn which helps the transmission efficiency of the ultrasonic wave between the test block and the waveguide [8].

$$
\varphi(\operatorname{deg})=70 \frac{\lambda}{D}=70 \frac{C}{D \cdot f}
$$




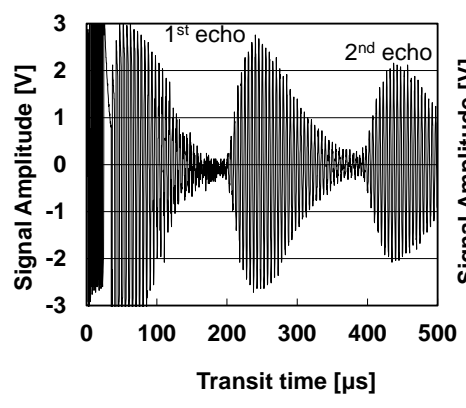

(a)

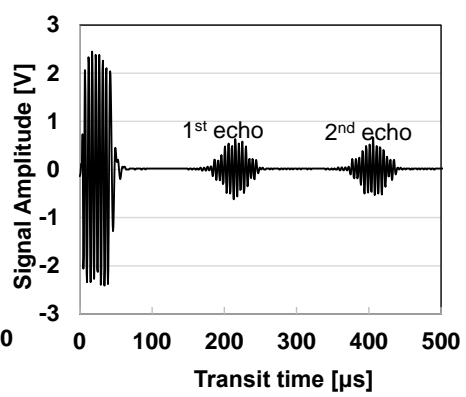

(b)

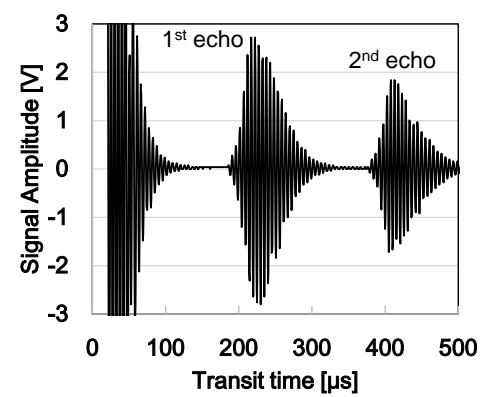

(c)

Figure 7. Received signal from the edge of a $500 \mathrm{~mm}$ long waveguide using 3 types of ultrasonic sensors. (a) PZT; (b) EMAT; (c) Ni-sheet.

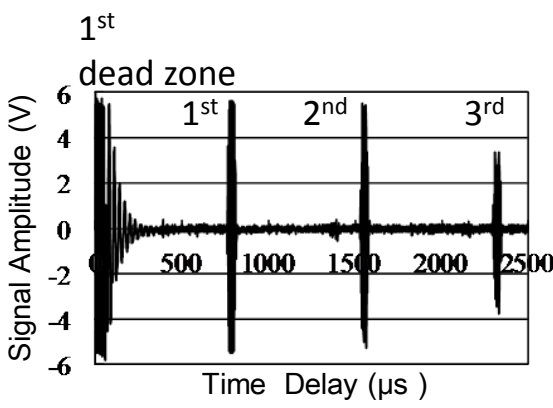

(a)

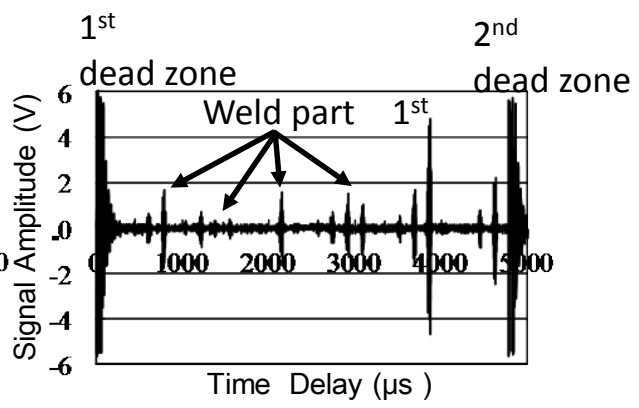

(b)

Figure 8. Received ultrasonic wave signal using a waveguide. (a) Length of waveguide = $2 \mathrm{~m}$; (b) Length of waveguide $=10 \mathrm{~m}$.

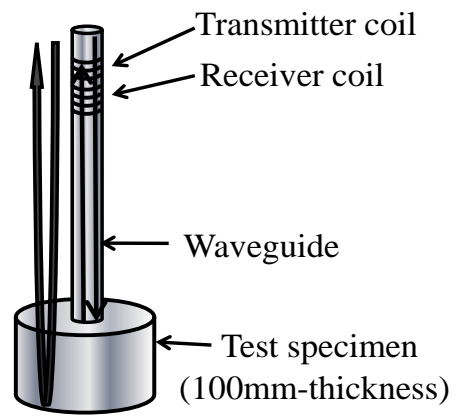

(a)

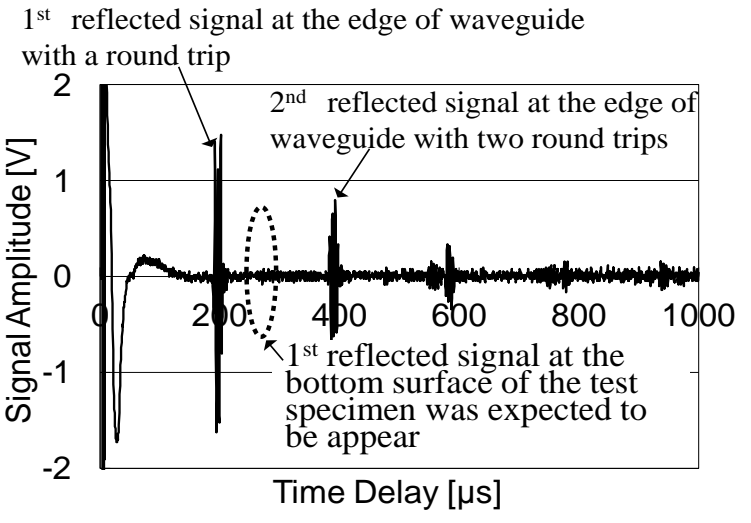

(b)

Figure 9. Reflected ultrasonic wave signal from the opposite side of a test specimen with only a waveguide. (a) Experimental setup; (b) Received ultrasonic wave signal using a waveguide.

$\varphi$ : directivity angle, $\lambda$ : wavelength, $D$ : diameter of the ultrasonic source, $f$ : frequency of the ultrasonic wave, $c$ : velocity of the ultrasonic wave.

Therefore, many acoustic horns were fabricated in order to increase the transmission efficiency. Figure 10(a) shows the case without an acoustic horn, Figure 10(b) shows the case using a cylindrical acoustic horn, and Figure 10(c) shows a conical acoustic horn.

Figure 11 is a summary of the results using two types of acoustic horns with many different specifications. In the case of the cylindrical shaped acoustic horn, the diameter ranged from $4 \mathrm{~mm}$ to $30 \mathrm{~mm}$ and the height was the same as the wavelength of the guide wave of $20 \mathrm{~mm}$. In the case of the conical-shaped acoustic horn, the diameter ranged from $4 \mathrm{~mm}$ to $30 \mathrm{~mm}$ and the height ranged from a half wavelength to a wavelength. The scale 


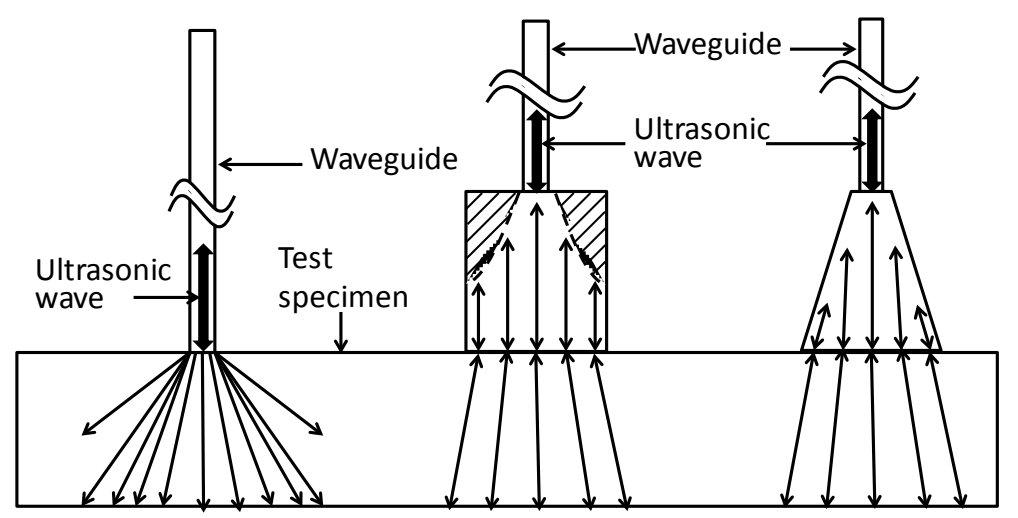

(a)

(b)

(10)

Figure 10. Shapes of different acoustic horns. (a) Without acoustic horn; (b) Stepped horn; (c) Conical horn.

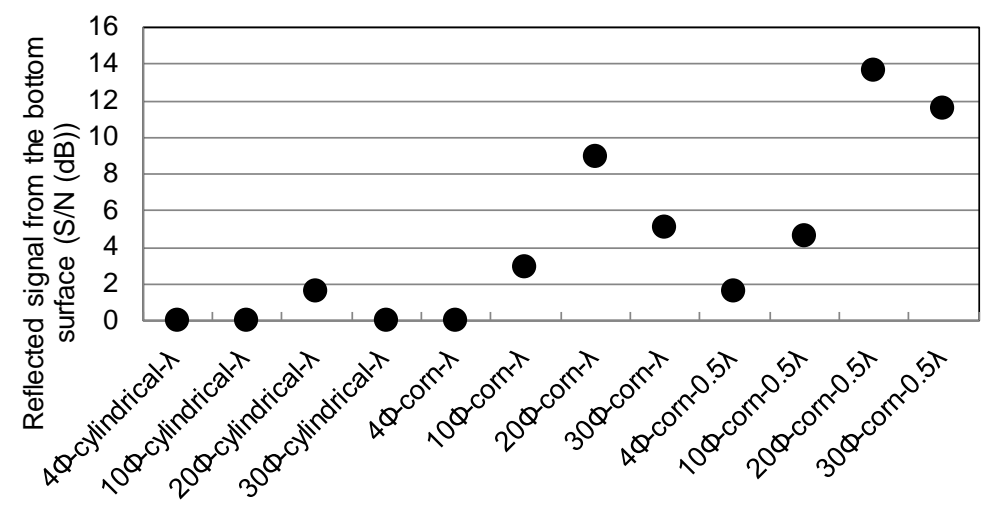

Figure 11. Relationship between ultrasonic wave signal strength $(\mathrm{S} / \mathrm{N})$ reflected at the bottom surface of the test block and acoustic horn.

of the horizontal axis number from 1 to 4 shows the results of the cylindrical horn and the scale of the horizontal axisnumber from 5 to 10 shows the results of the conical horn. The scale of the vertical axis shows the received signal amplitude $(\mathrm{S} / \mathrm{N})$ reflected at the bottom surface of the test block due to the difference in the acoustic horn specifications. The best signal was obtained using the conical acoustic horn with a $20 \mathrm{~mm}$ diameter and 0.5 wavelength height.

Figure 12(a) shows the reflected ultrasonic wave signal using a cylindrical horn of 20-mm diameter and a half-wavelength height. Figure 12(b) shows the reflected ultrasonic wave signal using a conical horn of 20-mm diameter and a half wavelength height. The received signal reflected on the opposite surface of the test specimen, when the cylinder horn was used, was much lower than that of the conical horn. It indicated that the conical horn was better that the cylindrical horn.

\subsection{Influence of Increasing Temperature}

The test block was heated to confirm whether the trial ultrasonic sensing system was useful. Figure 13 shows the experimental system. A steel bar of $500 \mathrm{~mm}$ length was used as the waveguide. Thermostat chambers, which can be heated to $1100^{\circ} \mathrm{C}$, were used in this experiment. The test block and the opposite side of the waveguide was installed in the thermostat chambers. As the experimental results, it was confirmed that the temperature at the place where the EMAT was attached was under $35^{\circ} \mathrm{C}$, even if the test specimen and the opposite side of the waveguide were heated to $500^{\circ} \mathrm{C}$ as shown in Figure 14. As a result, the ultrasonic sensing system was confirmed to be able to measure the received ultrasonic wave signal without being influenced by the temperature of the test block. 


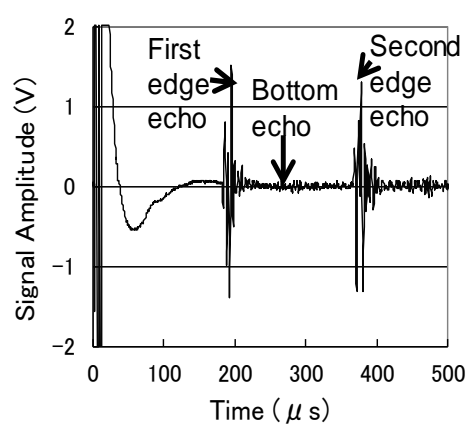

(a)

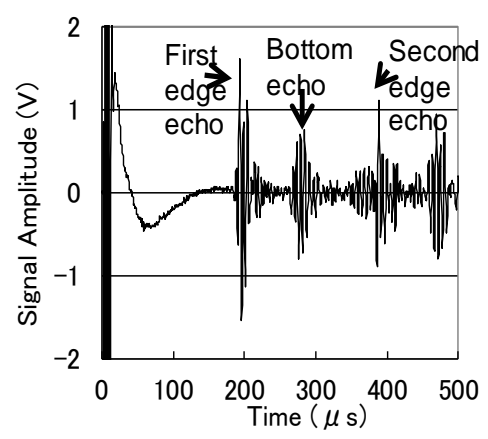

(b)

Figure 12. Reflected ultrasonic wave signal from the opposite side of the test specimen using a horn. (a) Without acoustic horn; (b) With conical horn.

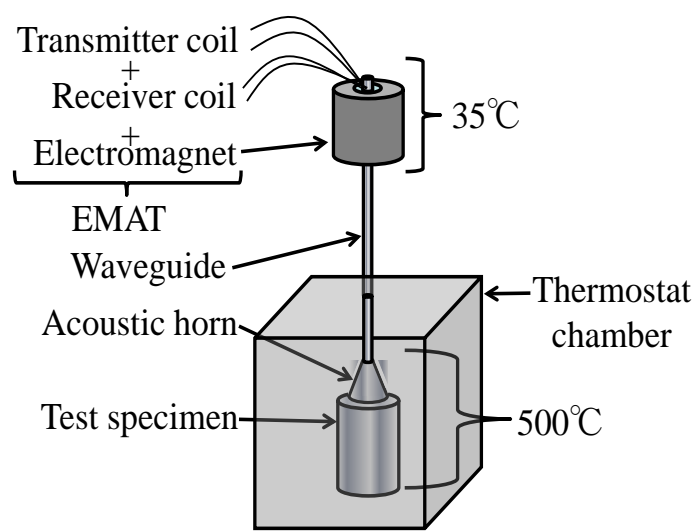

Figure 13. Experimental view when the test block was heated.

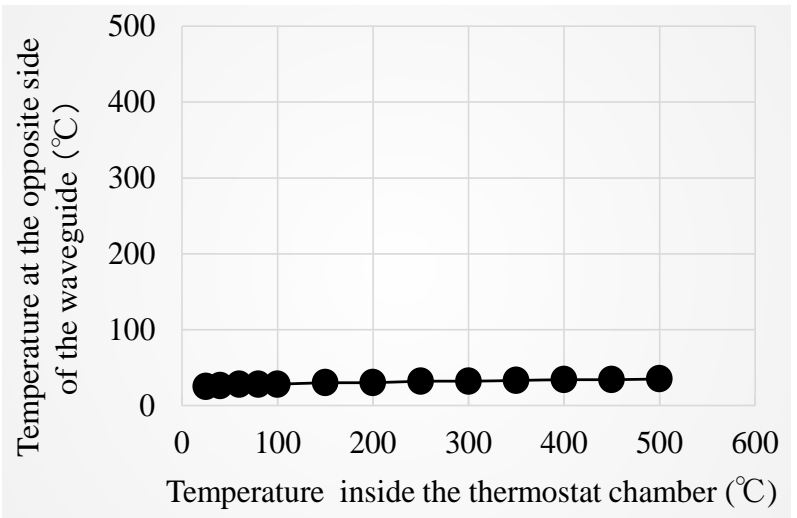

Figure 14. Relationship of temperature between edge (A) and edge (B).

\subsection{Influence of Bent Part}

When we tried to apply this technique to a structure in the field, it was difficult to use only a straight waveguide. The influence of the bent part of the waveguide as shown in Figure 15(a) was then evaluated. As a result, if the curvature radius is under $20 \mathrm{~mm}$, the reflected signal at the bend was clearly detected as shown in Figure 15(b). The influence of the relationship between the reflected signal at the bend, the curvature radius, and the bending angle was evaluated. Figure 15(c) shows examples of the waveguides with the same curvature radius and different bending angles. It was confirmed that it was independent of the bending angle if the curvature radius was over $20 \mathrm{~mm}$. 


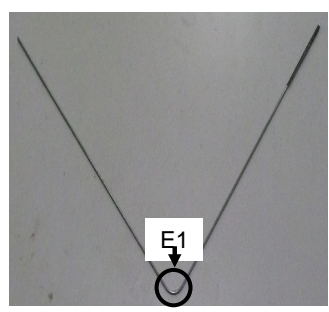

(a)

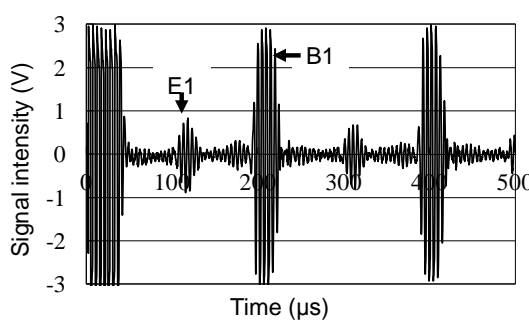

(b)

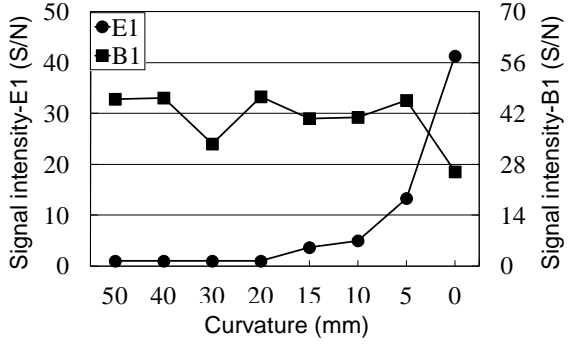

(10)

Figure 15. Influence of the bent part of the waveguide. (a) Bent part of a waveguide; (b) Reflected signal at the bent part; (c) Relation between B1 and E1t.

\section{Conclusion}

An ultrasonic sensing system using a long waveguide, a conical acoustic horn, and an acoustic horn based on Snell's law was used to perform a nondestructive inspection of a high temperature structure. First, we tried to determine the proper ultrasonic sensor by comparing 3 types of ultrasonic sensors. As a result, the ultrasonic sensor to drive a thin Ni sheet attached to the side surface of the steel bar using a dynamic and static magnetic field was selected. The specification and drive condition of the steel bar for a waveguide were then determined using a steel bar with a $0.5 \mathrm{~mm}-6.0 \mathrm{~mm}$ diameter. The results were a $2 \mathrm{~mm}$ diameter and $200 \mathrm{kHz}$. Next, the acoustic horn of 20-mm diameter and half wavelength height was selected to transmit and receive an ultrasonic wave with a good efficiency. The reflected signal at the bottom surface of the test specimen was confirmed using these systems. Next, the influence of the waveguide was investigated. If the bend in the waveguide becomes sharp, the reflected signal at the part will be detected. However, it was also confirmed that the reflected signal at the bend could not be detected if the curvature radius was over $20 \mathrm{~mm}$. Finally, the sensor part was confirmed to be driven at room temperature based on a high temperature experiment. Although the performance must be improved, these results indicated that a new nondestructive inspection system could be possible for use with a high temperature structure.

\section{Acknowledgements}

This study was partially supported by a grant-in-aid for Scientific Research from the Ministry of Education Culture, Sports, Science and Technology (C26420032).

\section{References}

[1] Tittmann, B.R. (2003) A Novel Technique with a Magnetostrictive Transducer for in Situ Length Monitoring of a Distant Specimen. Ultrasonic Nondestructive Evaluation for Material Science and Industries, 456, 73-78.

[2] Tittmann, B.R. and Aslan, M. (1999) Ultrasonic Sensors for High Temperature Applications. Japanese Journal of Applied Physics, 38, 3011-3013. http://dx.doi.org/10.1143/JJAP.38.3011

[3] Thompson, R.B. (1973) A Model for the Electromagnetic Generation and Detection of Rayleigh and Lamb Wave. IEEE Transaction on Sonics and Ultrasonic, 20, 340-346. http://dx.doi.org/10.1109/T-SU.1973.29770

[4] Yamasaki, T. (1999) Generation and Detection of Longitudinal Wave in Steel Wires by Electromagnetic Acoustic Transducers. Transactions of the Japan Society of Mechanical Engineers Series A, 65, 1038-1043. http://dx.doi.org/10.1299/kikaia.65.1038

[5] Hirao, M. and Ogi, H. (2003) EMATS for Science and Industry. Kluwer Academic Publishers, Berlin. http://dx.doi.org/10.1007/978-1-4757-3743-1

[6] Ogi, H., Goda, E. and Hirao, M. (2003) Increase of Efficiency of Magnetostriction SH-Wave EMAT by Angled Bias Field. Japanese Journal of Applied Physics, 42, 3020-3024. http://dx.doi.org/10.1143/JJAP.42.3020

[7] Hirao, M., Ogi, H. and Yasui, H. (2001) Contactless Measurement of Bolt Axial Stress Using a Shear-Wave Electromagnetic Acoustic Transducer. NDT \& E International, 34, 179-183. http://dx.doi.org/10.1016/S0963-8695(00)00055-4

[8] Hashiba, K. (1994) Power Flow in Solid Horns for Ultrasonics. Transactions of the Japan Society of Mechanical Engineers Series A, 60, 2871-2876. http://dx.doi.org/10.1299/kikaia.60.2871 
Scientific Research Publishing (SCIRP) is one of the largest Open Access journal publishers. It is currently publishing more than 200 open access, online, peer-reviewed journals covering a wide range of academic disciplines. SCIRP serves the worldwide academic communities and contributes to the progress and application of science with its publication.

Other selected journals from SCIRP are listed as below. Submit your manuscript to us via either submit@scirp.org or Online Submission Portal.
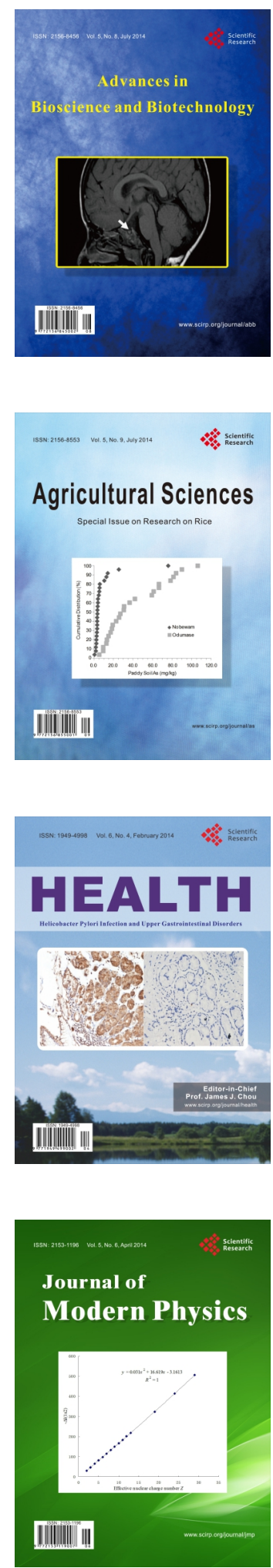
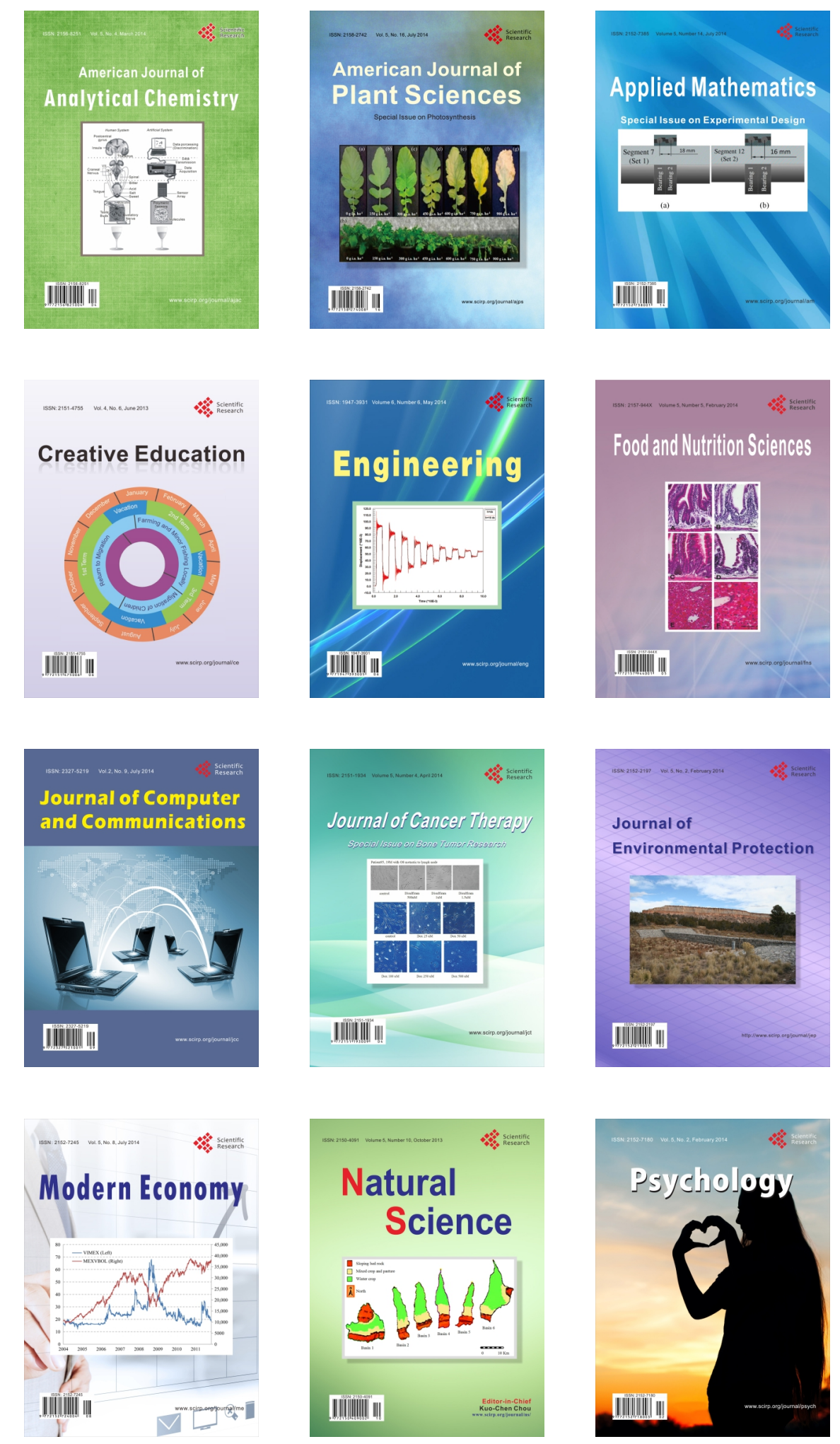